\title{
A multi-channel current-mode CMOS optical receiver array for active optical HDMI cables
}

\author{
Seung Hoon Kim ${ }^{1,2 a)}$, Jihye Shin ${ }^{2}$, Jinju Lee ${ }^{2}$, Xiao Ying ${ }^{2}$, \\ Hanbyul Choi ${ }^{2}$, Kyungmin Lee ${ }^{2}$, Chaerin Hong ${ }^{2}$, \\ Sang-Bock Cho ${ }^{1}$, and Sung Min Park ${ }^{2 b)}$ \\ ${ }^{1}$ School of Electrical Engineering, University of Ulsan, Ulsan, Korea \\ 2 Department of Electronics Engineering, Ewha Womans University, Seoul, Korea \\ a)pcs0879@nate.com \\ b)smpark@ewha.ac.kr, Corresponding Author
}

\begin{abstract}
This paper introduces a four-channel optical receiver array implemented in a standard 1P4M $0.18 \mu \mathrm{m}$ CMOS technology for the applications of active optical HDMI cables. Each channel consists of a current-mirror transimpedance amplifier, a five-stage differential limiting amplifier, and an output buffer, demonstrating $81-\mathrm{dB} \Omega$ transimpedance gain, 1.8-GHz bandwidth even with $0.5-\mathrm{pF}$ photodiode capacitance, $-18.3-\mathrm{dBm}$ optical sensitivity for $10^{-12}$ BER \& 0.6-A/W responsivity, and $51.7-\mathrm{mW}$ power dissipation from a single $1.8-\mathrm{V}$ supply. The whole chip occupies the area of $1.35 \times 2.46 \mathrm{~mm}^{2}$ including pads. The measured eye-diagrams of the array confirms wide and clear eye-openings up to $4.0-\mathrm{Gb} / \mathrm{s}$ operations.
\end{abstract}

Keywords: active optical cables, CMOS, current mirror, limiting amplifier, transimpedance amplifier

Classification: Integrated circuits

\section{References}

[1] J. Lee, J. Shin and S. Park: J. of IEEK SD-49 [8] (2012) 22.

[2] S. Shim and S. Park: J. of IEEK SD-43 [10] (2006) 1.

[3] J. Shin, J. Lee and S. Park: 2012 SoC Conference (2012).

[4] Y. Wang and R. Raut: IEEE ISCAS 2005 (2005) 1601. DOI:10.1109/ISCAS.2005. 1464909

[5] J. Ramirez-Angulo, M. S. Sawant, R. G. Carvajal and A. J. Lopez-Martin: IEEE ISCAS 2006 (2006) 2833. DOI:10.1109/ISCAS.2006.1693214

[6] J. Yun, M. Seo, B. Choi, J. Han, Y. Eo and S. M. Park: IEEE ISSCC 2009 (2009) 102. DOI:10.1109/ISSCC.2009.4977328

[7] J. Han, B. Choi, K. Park, W. S. Oh and S. M. Park: IEEE A-SSCC (2007) 156. DOI:10.1109/ASSCC.2007.4425754

[8] S. Goswami, J. Silver, T. Copani, C. Wenjian, H. J. Barnaby, B. Vermeire and S. Kiaei: ISSCC 2009 (2009) 100. DOI:10.1109/ISSCC.2009.4977327

[9] B. Razavi: Design of Integrated Circuits for Optical Communications (McGraw Hill, 2003).

[10] S. Galal and B. Razavi: IEEE ISSCC Dig. (2003) 188. DOI:10.1109/JSSC.2003. 818567 


\section{Introduction}

Recently, optical transports have been paid significant attention, which includes not only short-distance optical links for LAN and SAN, but also very short-reach optical links such as board-to-board and chip-to-chip interconnects. In particular, gigabit optical interface can be applied to consumer electronics e.g. HDMI (high definition multimedia interface), DVI (digital visual interface) [1]. Optical links provide a number of advantages over typical copper-based electrical links including much less signal attenuation, power dissipation, EMI (electromagnetic interference), etc. Therefore, attempts have been conducted to replace even the very short reach HDMI data transport systems with active optical cables for maintaining high signal qualities [2].

For this purpose, it should be preceded to develop low-cost optical receiver solutions which yield wide-bandwidth, low-noise, and low power consumption characteristics. Especially, the total receiver system performance is mainly determined by its front-end circuits including transimpedance amplifier (TIA), and limiting amplifier (LA). Thereby, we realize a four-channel low-power optical receiver array chip in a $0.18 \mu \mathrm{m}$ CMOS technology. Each channel consists of a TIA, a LA, and an output buffer, demonstrating the link speed of up to 4.0-Gb/s. The TIA takes the configuration of current-mode topology which is the modified current-mirror $(\mathrm{CM})$ input stage with local feedback $[3,4,5]$. Then, the TIA achieves high operation speed with no equalizer circuit [6].

Previously, Y. Wang et al. [4] proposed the modified CM TIA configuration, achieving $2.5-\mathrm{Gb} / \mathrm{s}$ operations which were however only simulated results. J. Yun et al. [6] reported a $4-\mathrm{Gb} / \mathrm{s}$ receiver utilizing a conventional CM TIA, yet providing electrically measured results for a single-channel receiver. J. Han et al. [7] exploited a common-gate current-mode technique to realize a dual-channel $2.5-\mathrm{Gb} / \mathrm{s}$ optical receiver. Also, it demonstrated electrically measured operations only. S. Goswami et al. [8] proposed a modified regulated cascode TIA, obtaining very low-power 5$\mathrm{Gb} / \mathrm{s}$ operations. However, only a single-channel TIA was electrically measured. In this paper, the modified CM TIA is incorporated to realize a 4-channel optical receiver array operating up to $4-\mathrm{Gb} / \mathrm{s}$ per channel for the applications of active optical HDMI cables.

Section II describes the architecture of the proposed multi-channel optical receiver circuit and the circuit operations of its main building blocks. In Section III, the measured results of the proposed chip are demonstrated. Finally, conclusion is followed.

\section{Circuit description}

\subsection{Current-Mirror TIA}

Fig. 1 shows the block diagram of the proposed current-mode optical receiver, where the CM-TIA represents the current-mirror TIA and the LA represents the limiting amplifier with a $50 \Omega$ output buffer.

The TIA exploits a single-ended current-mirror input stage, a passive LPF (lowpass filter) for single-to-differential conversion, a two-stage differential commonsource amplifier for gain-boosting, and a $70 \Omega$ output buffer to facilitate the loading 


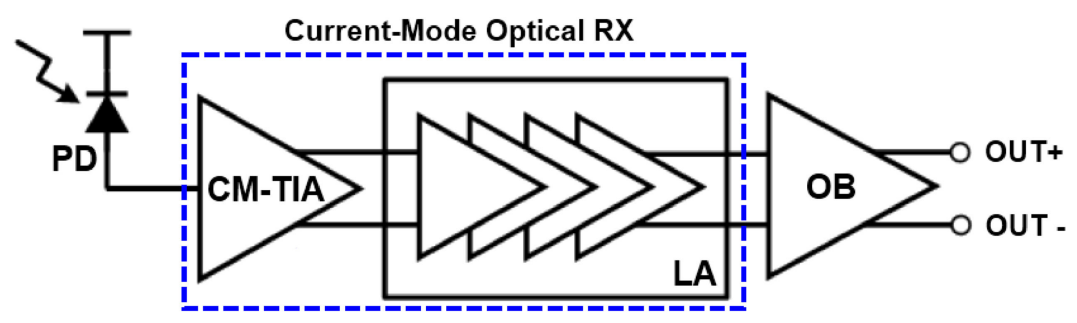

Fig. 1. Block diagram of the proposed current-mode optical receiver circuit.

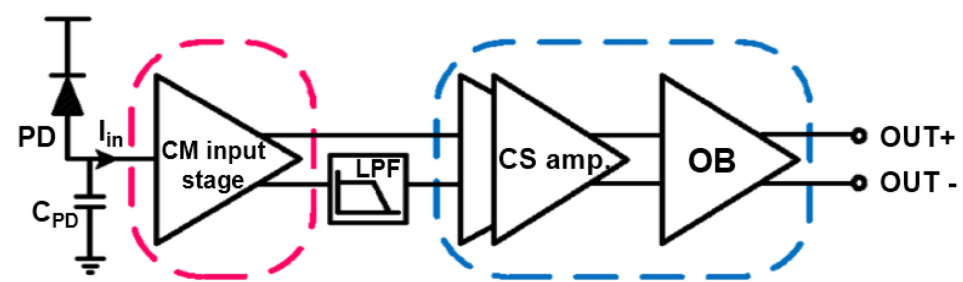

Fig. 2. Block diagram of the CM-TIA.

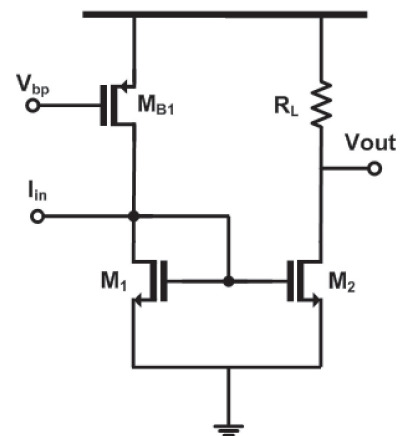

(a)

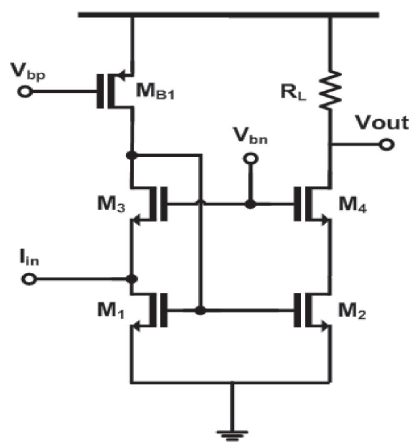

(b)

Fig. 3. Schematic diagrams of (a) the conventional current-mirror input stage, and (b) the modified current-mirror circuit.

effect of the high-gain LA (shown in Fig. 2). The CM-TIA operates as a currentmode, thereby providing low input impedance at the input node. Hence, it can isolate the large input parasitic capacitance effectively, and thus achieve wide bandwidth.

The half-rate phase detector automatically yields the demuxed outputs, Dout1 and Dout2. However, a MUX circuit is employed at the end of the phase detector, so as to acquire the outputs of the same speed as the inputs for testing purposes.

The passive LPF converts the single-ended outputs of the CM-TIA to differential signals for following stages, so that the characteristics of common-mode noise rejection is enhanced. The next two-stage common-source amplifiers employs source-degeneration techniques to achieve high gain and wide bandwidth simultaneously [9].

Fig. 3 depicts the schematic diagrams of both CM input stages: (a) conventional CM input, (b) modified CM input. According to small signal analysis, the conventional $\mathrm{CM}$ input stage provides the input resistance, the transimpedance gain, the bandwidth, and the equivalent noise spectral density as shown below [6]. 
Here, it is assumed that the aspect ratios of $M_{1}$ and $M_{2}$ are the same, and that the input capacitance $\left(\mathrm{C}_{\mathrm{IN}}\right)$ represents the sum of the gate capacitance of $\mathrm{M}_{2}$ and all parasitic capacitances at the gate and drain nodes of $\mathrm{M}_{1}$. It is noted that the input resistance of the conventional CM input stage can be reduced by controlling $\mathrm{g}_{\mathrm{m} 1}$, and thus the bandwidth can be extended. However, a design tradeoff between transimpedance gain and bandwidth occurs in accordance with the load resistance $\mathrm{R}_{\mathrm{L}}$, which mandates the optimum value of $\mathrm{R}_{\mathrm{L}}$.

For the case of noise characteristics, the conventional CM input stage needs to boost $\mathrm{g}_{\mathrm{m} 2}$ in order to reduce the effect of large input capacitance $\left(\mathrm{C}_{\mathrm{IN}}\right)$. It also leads to high transimpedance gain for similar DC power consumption.

Yet, the large value of $g_{m}$ requires either wide transistors or large bias currents. The former may limit the bandwidth because of the enlarged parasitic capacitance, while the latter increases the power dissipation. Therefore, the value of $g_{m}$ should be judiciously selected for design optimization.

Fig. 3(b) shows the modified CM input stage of which small signal analysis yields the input resistance, the transimpedance gain, the bandwidth, and the equivalent noise spectral density as shown below [3].

$$
\begin{aligned}
& Z_{I N}(0)=r_{01}\left\|r_{0 B 1}\right\| \frac{1}{g_{m 1}} \cong \frac{1}{g_{m 1}} \\
& Z_{T}(0)=\frac{V_{O U T}}{I_{I N}}=\frac{(W / L)_{2}}{(W / L)_{1}}\left(R_{L} \| r_{02}\right) \cong R_{L} \\
& f_{-3 d B} \cong \frac{1}{2 \pi \cdot R_{L}\left(C_{g d 2}+C_{L}\right)} \\
& \overline{i_{e g}^{2}} \cong \frac{4 k T T}{g_{m 2}} w^{2}\left(C_{P D}+C_{I N}\right)^{2}+4 k T T\left(g_{m 1}+g_{m 51}\right) \\
& Z_{I N}(0) \cong \frac{1}{g_{m 1} g_{m 3}\left(r_{O B 1} \| r_{O 3}\right)} \cong \frac{1}{g_{m 1} A_{f}} \\
& Z_{T}(0) \cong \frac{g_{m 2}}{g_{m 3}}\left(R_{L} \| r_{o 2}\right)=\frac{(W / L)_{2}}{(W / L)_{1}} g_{m 3}\left(R_{L} \| r_{o 2}\right) \\
& f_{-3 d B} \cong \frac{1}{2 \pi\left\{\frac{\left(C_{P D}+C_{I N}\right)}{g_{m 1} A_{f}}+R_{L}\left(C_{g d 4}+C_{g d 4}+C_{x}\right)\right\}} \\
& \overline{i^{2}{ }_{e g}} \cong \overline{i_{d 1}^{2}}+\overline{i^{2} d B 1}+\frac{\overline{i_{d 2}}+\overline{i^{2} R L}}{g^{2} m 2} \\
& \times\left\{\frac{1}{r_{o B 1}^{2}}+\frac{1}{g^{2}{ }_{m 3} r_{o 3}^{2}}\left(\frac{1}{r_{o 1}^{2}}+\omega^{2} C_{I N t o t}^{2}\right)+\omega^{2} C_{d 3}^{2}\right\} \\
& \cong \overline{i_{d 1}^{2}}+\overline{i_{d B 1}^{2}}+\overline{i_{d 2}^{2}} \omega^{2} C_{d 3}^{2} \\
& =4 k T T\left(g_{m 1}+g_{m B 1}\right)+\frac{4 k T T}{g_{m 2}} \omega^{2} C_{d 3}^{2}
\end{aligned}
$$

In Eq. (5), it is clearly seen that the input impedance of the modified CM input stage is Af (feedback gain) times lower than that of the conventional one. This reduces the time constant occurred at the input node and hence the bandwidth of the whole circuit is expanded. The transimpedance gain in Eq. (6) is the same as that in 
Eq. (2). The bandwidth in Eq. (7) can be wider than Eq. (3) because the large input capacitance including $\mathrm{C}_{\mathrm{PD}}$ and $\mathrm{C}_{\mathrm{IN}}$ is effectively isolated from the bandwidth determination by the amount of the local feedback gain. $C_{X}$ in Eq. (7) represents the input capacitance. Also, the effect of the input capacitance is no longer present in the equivalent noise current spectral density of Eq. (8).

Instead, the drain capacitance of $\mathrm{M}_{3}\left(\mathrm{C}_{\mathrm{d} 3}=\mathrm{C}_{\mathrm{gd} 1}+\mathrm{C}_{\mathrm{dbB} 1}+\mathrm{C}_{\mathrm{gd} 3}+\mathrm{C}_{\mathrm{db} 3}\right)$ appears, but it is much smaller than the input capacitance $\left(\mathrm{C}_{\mathrm{INtot}}=\mathrm{C}_{\mathrm{PD}}+\mathrm{C}_{\mathrm{gd} 1}+\right.$ $\mathrm{C}_{\mathrm{db} 1}+\mathrm{C}_{\mathrm{gs} 3}+\mathrm{C}_{\mathrm{sb} 3}$ ). Conclusively, the input resistance of the modified CM input stage can be reduced by controlling the value of $A_{f}$ and $g_{m 1}$, and thus the wider bandwidth can be achieved. Yet, the transconductance $\left(\mathrm{g}_{\mathrm{m} 1}\right)$ of $\mathrm{M}_{1}$ should be cautiously selected for the optimized design between input resistance, bandwidth, and noise current spectral density.

\subsection{Limiting amplifier}

Fig. 4 illustrates the block diagram of the gain-scaling limiting amplifier (LA) which comprises five-stage amplifiers, an offset cancellation circuit, and an output buffer [7, 10]. The first two gain-cells take the configuration of Cherry-Hooper differential pair to mitigate the loading effect of the CM-TIA, whereas the next three gain-cells exploit source-degeneration technique so that the bandwidth maintains for $2.5-\mathrm{Gb} / \mathrm{s}$ operation speed per channel. HSPICE simulations were conducted for the whole optical receiver by utilizing the design parameters of a standard $0.18-\mu \mathrm{m}$ CMOS technology. Fig. 5 shows the frequency responses in which the CM-TIA achieves $54.3-\mathrm{dB} \Omega$ transimpedance gain and $2.6-\mathrm{GHz}$ bandwidth, while the optical receiver obtains $81-\mathrm{dB} \Omega$ trans-impedance gain and 1.8$\mathrm{GHz}$ bandwidth. Fig. 5(a) depicts the eye-diagrams of the proposed CM-TIA for $100 \mu \mathrm{A}_{\mathrm{pp}}$ 2.5-Gb/s $2^{31}$-1 PRBS input data stream, while Fig. 5(b) shows the eye-

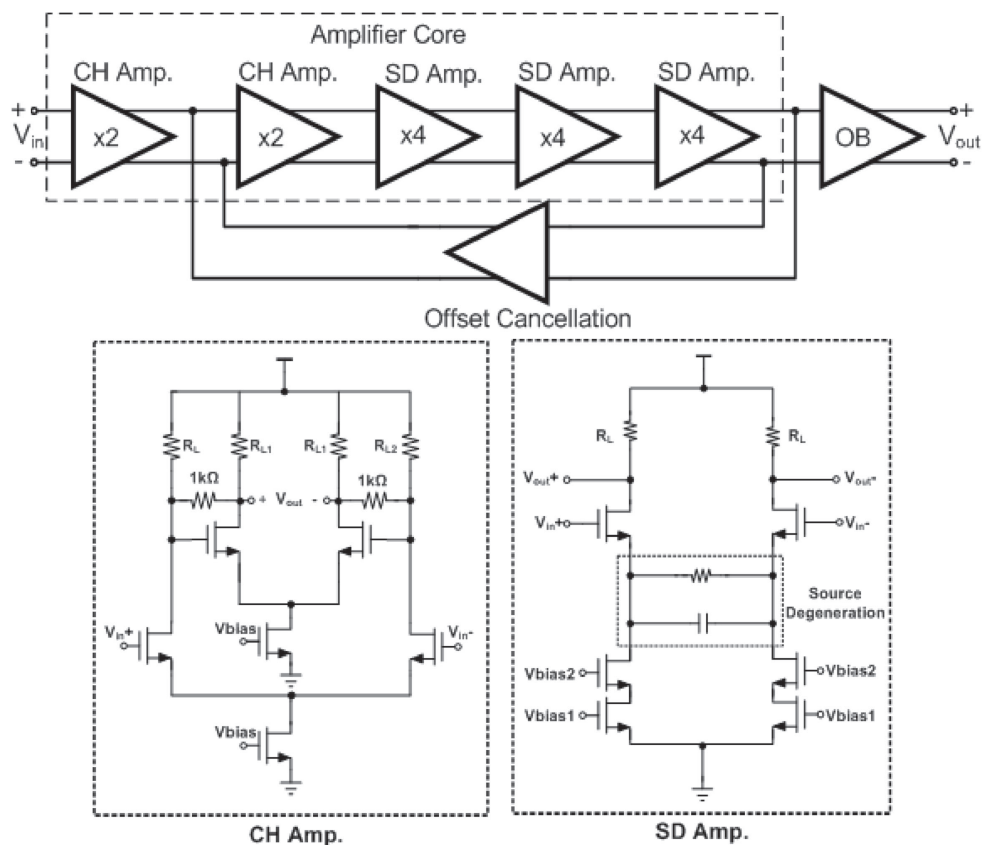




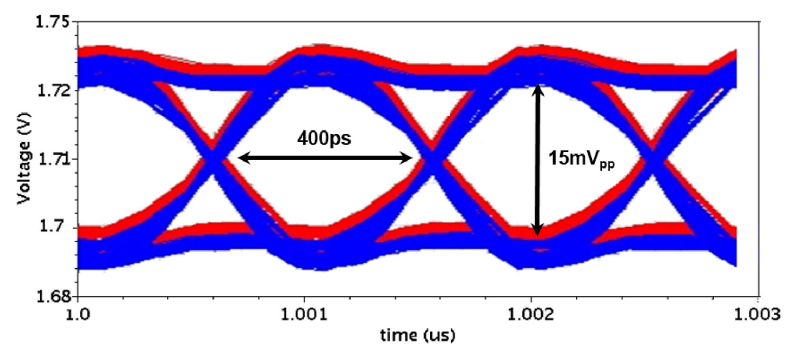

(a)

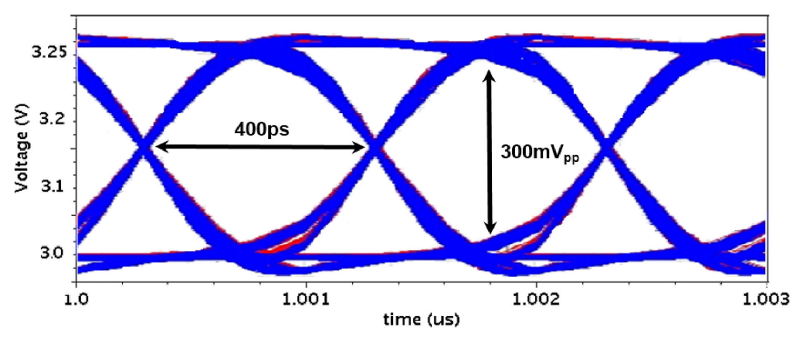

(b)

Fig. 5. Simulated eye-diagrams of (a) the proposed CM-TIA, and (b) the optical RX for $2.5-\mathrm{Gb} / \mathrm{s} 2^{31}-1$ PRBS input data stream.

diagrams of the whole receiver in which the single-ended amplitude reaches $\sim 300 \mathrm{mV}_{\mathrm{pp}}$.

\section{Chip fabrication and measured results}

Test chips of the proposed 4-channel optical receiver (RX) array circuit were fabricated by using a standard $0.18-\mu \mathrm{m}$ 1P4M CMOS process. Fig. 6 depicts the whole layout of the proposed 4-channel array chip, where the chip occupies the area of $1.35 \times 2.46 \mathrm{~mm}^{2}$ including I/O pads. Fig. 7 illustrates the test setup for the proposed 4-channel receiver array, where POF (plastic optical fiber) is utilized as the transmission medium from the TX. Fig. 8 demonstrates the optically measured eye-diagrams for $2.5-\mathrm{Gb} / \mathrm{s}$ and $3.5-\mathrm{Gb} / \mathrm{s} 2^{31}-1$ PRBS input signals (with $\mathrm{i}_{\text {in }}=50 \mu \mathrm{A}$ or $\mathrm{P}_{\text {in }}=-12 \mathrm{dBm}$ ). Each channel yields very similar performance. Here, it should be noted that the TX emits the optical signals with relaxation oscillation and its effect is completely diminished at the RX outputs. It may be attributed to the POF characteristics and the limited bandwidth of the RX. The output swing of the RX is measured to be $\sim 500 \mathrm{mV}_{\mathrm{pp}}$ single-ended. Fig. 9 also demonstrated the optically measured eye-diagrams of the 4-channel $\mathrm{RX}$ array with the input power of $-1.6 \mathrm{dBm}$ for $1.25-\mathrm{Gb} / \mathrm{s}, 2.5-\mathrm{Gb} / \mathrm{s}, 3.125-\mathrm{Gb} / \mathrm{s}$, and $4.0-\mathrm{Gb} / \mathrm{s}$

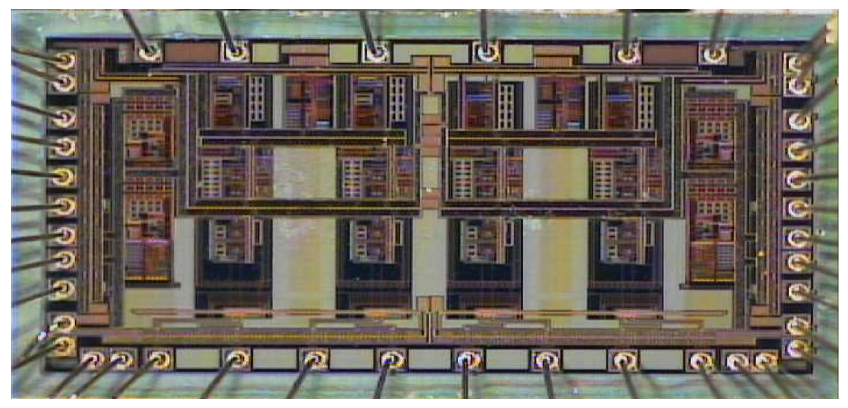

Fig. 6. Chip microphotograph of the proposed 4-ch. optical RX. 


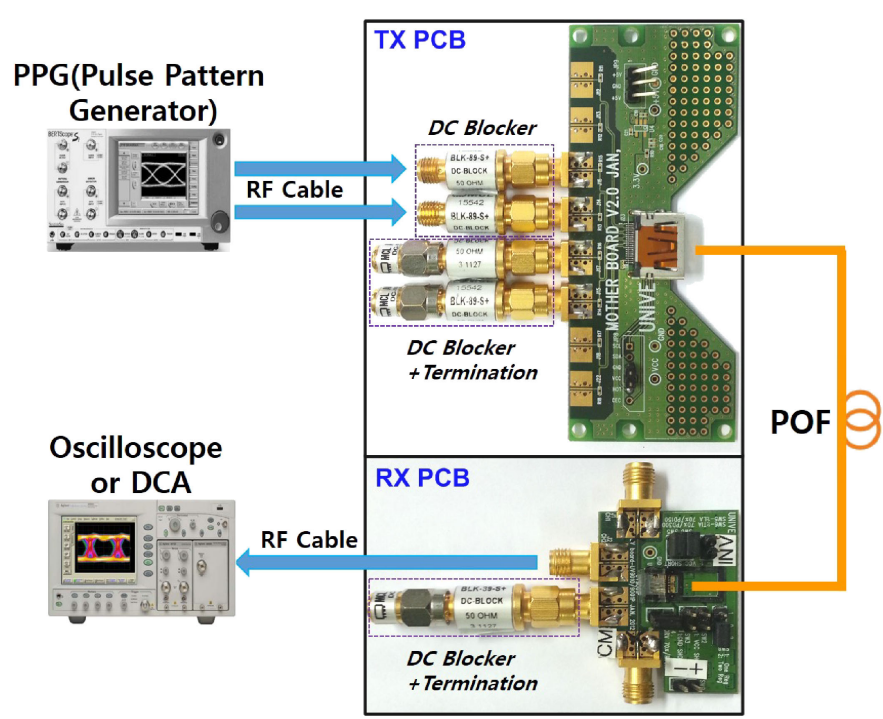

Fig. 7. Test setup for the proposed 4-ch. optical RX.

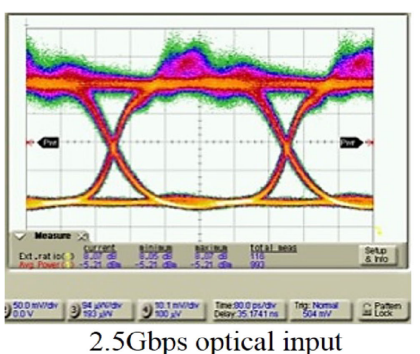

2.5Gbps optical input

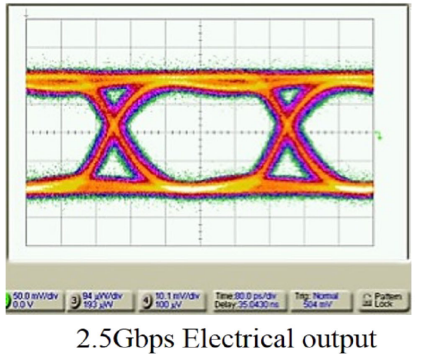

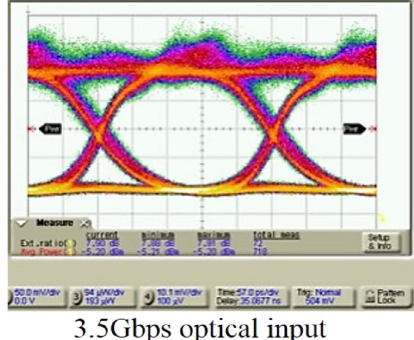

$3.5 \mathrm{Gbps}$ optical input

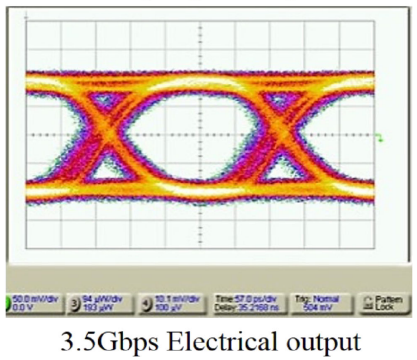

Fig. 8. Optically measured eye-diagrams of the proposed TX and RX for $2.5-\mathrm{Gb} / \mathrm{s} \& 3.5-\mathrm{Gb} / \mathrm{s} 2^{31}-1$ PRBS input data stream (when $\left.\mathrm{i}_{\text {in }}=50 \mu \mathrm{A}\right)$.

$2^{31}-1$ PRBS, respectively. It is clearly seen that the proposed RX array achieves wide and clear eye-openings for up to $4.0-\mathrm{Gb} / \mathrm{s}$ operations.

The proposed RX array is implanted in an optical module and tested in a practical environment. Fig. 10 shows the HDMI demo video still image provided through the utilized active optical HDMI cables.

DC measurements reveals that each channel of the proposed chip dissipates $51.7 \mathrm{~mW}$ (including output buffer) from a single $1.8 \mathrm{-V}$ supply, in which the CMTIA consumes $10.9 \mathrm{~mW}$ and the gain-scaling LA dissipates $40.9 \mathrm{~mW}$.

The performance of the proposed 4-channel RX array chip is summarized in Table I, along with the comparison of the prior arts realized in CMOS process. It is clearly seen that this work provides optically measured data up to $4.0-\mathrm{Gb} / \mathrm{s}$ operations for 4-channels with comparable sensitivity and power consumption characteristics among those recently published current-mode TIAs. 


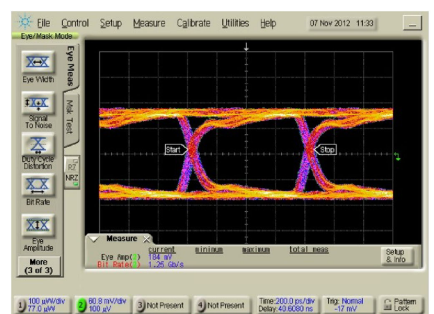

(a) $1.25 \mathrm{~Gb} / \mathrm{s}$

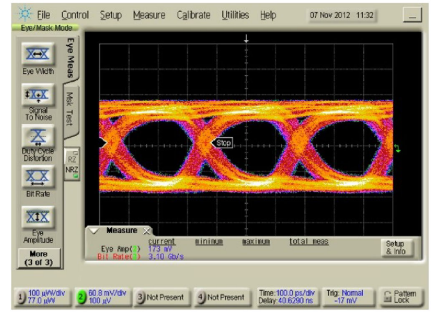

(c) $3.125 \mathrm{~Gb} / \mathrm{s}$

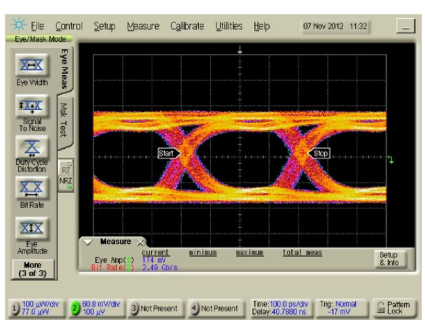

(b) $2.5 \mathrm{~Gb} / \mathrm{s}$

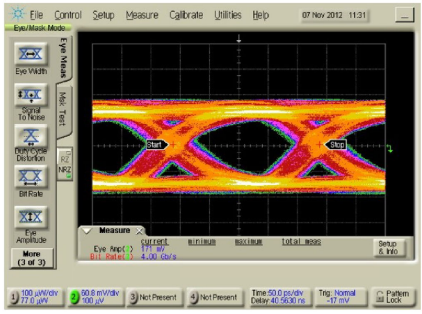

(b) $4.0 \mathrm{~Gb} / \mathrm{s}$

Fig. 9. Optically measured eye-diagrams of the proposed $\mathrm{RX}$ for (a) $1.25-\mathrm{Gb} / \mathrm{s}$, (b) $2.5-\mathrm{Gb} / \mathrm{s}$, (c) $3.125-\mathrm{Gb} / \mathrm{s}$, (d) $4.0-\mathrm{Gb} / \mathrm{s} 2^{31}-1$ PRBS input data streams (when $P_{\text {in }}=-1.6 \mathrm{dBm}$ ).
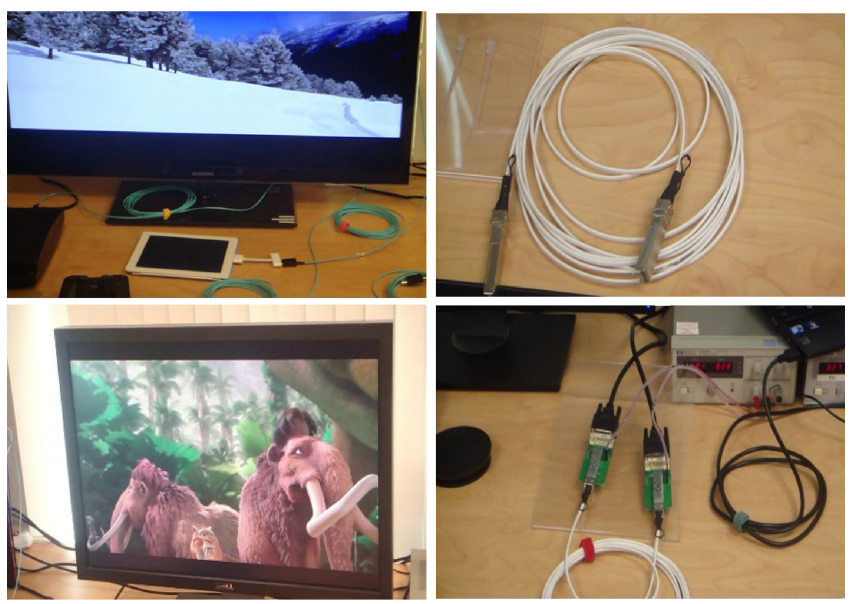

Fig. 10. HDMI demo video still images and the implemented optical module of optical RX array. (courtesy UNIVE Corp.)

Table I. Performance summary of the proposed TIA and comparison with the conventional current-mode TIAs.

\begin{tabular}{l|c|c|c|c|c|c}
\hline Parameters & {$[3]$} & {$[4]$} & {$[6]$} & {$[7]$} & {$[8]$} & This work \\
\hline Technology $(\mu \mathrm{m})$ & 0.18 & 0.18 & 0.18 & 0.18 & 0.13 & 0.18 \\
\hline Architecture & TIA & TIA & TIA/EQ/LA & TIA/LA & TIA & TIA/LA \\
\hline $\begin{array}{l}\text { Input } \\
\text { Configuration }\end{array}$ & $\begin{array}{c}\text { Modified } \\
\text { CM }\end{array}$ & $\begin{array}{c}\text { Modified } \\
\text { CM }\end{array}$ & CM & CG & RGC & $\begin{array}{c}\text { Modified } \\
\text { CM }\end{array}$ \\
\hline Measurements & electrical & simulated & electrical & electrical & electrical & optical \\
\hline No. of channel & 1 & 1 & 1 & 2 & 1 & 4 \\
\hline $\begin{array}{l}\text { Max. operation } \\
\text { speed }(\mathrm{Gb} / \mathrm{s}) / \mathrm{ch}\end{array}$ & 2.5 & 2.5 & 4.0 & 2.5 & 5.0 & 4.0 \\
\hline TZ gain $(\mathrm{dB} \Omega)$ & 58 & 82 & 108 & 87 & 52 & 81 \\
\hline $\begin{array}{l}\text { Sensitivity }(\mathrm{dBm}) \\
\text { for } 10^{-12} \mathrm{BER}\end{array}$ & - & -17 & -19 & -18 & -9.3 & -18.3 \\
\hline $\begin{array}{l}\text { Power dissipation } \\
(\mathrm{mW} / \mathrm{ch})\end{array}$ & 32 & 19.7 & 70 & 50 & 5 & 51.7 \\
\hline
\end{tabular}




\section{Conclusion}

A 4-channel optical receiver array is realized in a $0.18-\mu \mathrm{m}$ CMOS technology for HDMI active optical cables. Each channel provides multi-rate operation speeds (i.e. 4.0-Gb/s/ch in maximum). The proposed RX exploits the TIA with modified current-mirror input stage and the LA with gain-scaling configuration. Optically measured results of the proposed RX chip demonstrate large and clean eyediagrams up to $4.0 \mathrm{~Gb} / \mathrm{s}$. Hence, the proposed chip provides a potential for active optical interfaces including HDMI applications.

\section{Acknowledgments}

This research was supported by the MSIP (Ministry of Science, ICT and Future Planning), Korea, under the ITRC (Information Technology Research Center) support program (NIPA-2014-(H0301-14-1008)) supervised by the NIPA (National IT Industry Promotion Agency). Also, this work was supported by IDEC (EDA Tool). Finally, authors would like to thank Y. Kim and Dr. Y. S. Son from UNIVE Corp. for their technical support. 